DOI: 10.17117/na.2015.12.01.268

http://ucom.ru/doc/na.2015.12.01.268.pdf

Поступила (Received): 28.12.2015

\title{
Мясников Е.С.
}

\section{Конкурентоспособность предприятия и проблема} привлечения экономических ресурсов

\author{
Myasnikov E.S. \\ The competitiveness of businesses and the \\ problem of attracting economic resources
}

В статье рассматривается вопрос важности обновления и пополнения ресурсной базы хозяйствующего субъекта. Дана классификация ресурсов и обозначены пути их привлечения. Выделены типы стратегий привлечения ресурсов. Отдельное внимание уделено проблеме конкурентоспособности в соответствии с современными тенденциями глобализационных процессов. Обозначены перспективы эффективного функционирования хозяйствующего субъекта на разных этапах его жизненного цикла. Как следствие была выявлена особая роль привлечения ресурсов и влияние их на конкурентоспособность

Ключевые слова: ресурсы, привлечение ресурсов, конкурентоспособность, глобализация

\section{Мясников Евгений Сергеевич \\ Аспирант \\ Международный банковский институт \\ 2. Санкт-Петербург, Невский пр., 60}

\begin{abstract}
The article considers the importance of refreshing and replenishment the resource base of the enterprise. The classification of resources and ways of their involvement marked. Obtained types of strategies to attract resources. Special attention is paid to the problem of competitiveness in line with current trends of globalization processes. Also perspectives of the effective functioning of the economic entity at different stages of its life cycle highlighted there. As a result was revealed the special role of mobilizing resources and their influence on competitiveness
\end{abstract}

Key words: resources, resources attraction, competitiveness, globalization
Myasnikov Evgeniy Sergeevich Graduate
International banking institute
Saint-Petersburg, Nevsky ave., 60

Ресурсы являются важнейшим элементом внутренней среды предприятия, от которого в большей мере зависят его результаты и функционирование. Ресурсы - это элемент, без которого экономическая деятельность не возможна. Этот факт обусловливает большое значение для решения целого комплекса вопросов по привлечению ресурсов, введение необходимой ресурсной базы предприятия, использованию и воспроизводству ресурсов. Данные вопросы объединены с управлением, что в свою очередь говорит о том, что для каждого предприятия ресурсы выступают одним из объектов управленческой деятельности.

Любая модель управления, не зависимо от объекта управления, основывается на определенной концепции, которая выступает в роли системы идей, представлений, принципов, предопределяющих цель функционирования пред- 
приятия, механизмы взаимосвязи объекта и субъекта управления, характер отношений между отдельными частями его внутренней структуры, а также необходимое влияние внешней среды на развитие предприятия [4].

В соответствии с сегодняшними тенденциями, определяемыми, преимущественно, глобализационными процессами, привлечение внешних экономических ресурсов рассматривается как важнейший фактор развития всех хозяйствующих субъектов. Поскольку ни одно современное предприятие не может развиваться с опорой только на собственные силы, привлечение внешних экономических ресурсов становится важной управленческой задачей. В силу того, что в условиях глобализации расширяется состав источников и масштабы привлечения ресурсов, возникает необходимость в проведении тщательного анализа всех существующих возможностей с целью поиска оптимальных управленческих решений. Это требует не только разработки практического инструментария такой работы, но и теоретического осмысления сущности экономических ресурсов с позиции современности [9, с. 88].

Анализ современных подходов к исследованию экономических ресурсов показывает, что единой точки зрения на понимание их экономической сущности нет. Так, среди известных трактовок наибольшее распространение получил подход, в рамках которого экономические ресурсы понимают как средства в форме доходов и внешних поступлений, предназначенные для выполнения обязательств и осуществления расширенного воспроизводства [5, с. 83]. Часть исследователей определяют экономические ресурсы с позиции экономических отношений, возникающих при их формировании, распределении и использовании. Они предлагают считать экономическими ресурсами совокупность благ, связанных с формированием и использованием основных фондов и накоплений на общегосударственные цели и финансированием затрат самих предприятий [2, с. 37]. Более универсальным можно считать определение, согласно которому под экономическими ресурсами предлагается понимать все источники развития предприятия [6, с. 288].

Опираясь на такое понимание ресурсов, представляется рациональным выделить среди них ресурсы внутренние - образовавшиеся внутри предприятия в ходе финансово-хозяйственной деятельности и внешние - обращающиеся на рынках капитала и находящиеся в форме государственных ресурсов [3, с. 101-102].

Классификация внутренних ресурсов осуществляется на основе структурного деления производительного капитала в процессе кругооборота на постоянный и переменный капитал и добавленную стоимость. В качестве критерия выступает процесс воспроизводства стоимости, который может осуществляться в постоянном или расширенном масштабе. Если процесс осуществляется в расширенном масштабе, то производительный капитал увеличивается, что делает возможным формирование дополнительных ресурсов.

Критерием классификации внешних ресурсов выступает форма капитала, который вкладывается внешними участниками в развитие данного предприятия. Форма капитала может иметь вид ссудного капитала. На рынке капитала осуществляется долевое финансирование, которое преследует цель получения 
прибыли и прав на управление фирмой. Долговое финансирование осуществляется на рынке ссудного капитала и предполагает не вложение капитала в фирму, а лишь передачу его во временное пользование с целью прироста капитала. Внешние ресурсы обращаются на рынках капитала, и привлечение их в организацию осуществляется путем использования управленческих инструментов $[8$, с. 23].

Выделяют следующие типы стратегий привлечения финансовых средств:

- Внутренняя направленность подразумевает использование собственных средств, в то время как внешняя - использование заёмных (облигационные займы, банковские кредиты.

- Использование собственных средств фирмы. Как правило, менее прибыльная стратегия, применяемая на сложившихся устойчивых рынках.

- Стратегия объединения финансовых ресурсов фирм, используемая для освоения новых рынков.

- Аккумулирование всех доступных источников финансирования (как внутренних, так и внешних) для осуществления перспективных проектов в различных отраслях. Высокорентабельно, но связанно с определёнными рисками.

- Предоставление финансирования одним подразделениям за счет прибыли других подразделений (в рамках одного предприятия) [1].

Большинство хозяйствующих субъектов в настоящее время испытывают затруднения с тем, чтобы эффективно развиваться только за счет собственных ресурсов. Следовательно, они вынуждены все активнее обращаться к внешним источникам, которые дают возможность получать экономические ресурсы на привлекательных условиях.

Перспективы эффективного функционирования хозяйствующего субъекта на разных этапах его жизненного цикла определяются характером использования собственных средств и необходимостью привлечения заемных средств желаемого объема и на приемлемых условиях. В современных условиях это нередко приобретает форму управленческого воздействия, направленного на поддержание оптимального соотношения между собственным и заемным капиталом. Именно это соотношение в купе с эффективностью и качеством привлекаемых ресурсов, помимо прочего, оказывает серьезное влияние на конкурентоспособность предприятия, что в свою очередь является одним из основополагающих объектов исследования в экономической науке.

Конкурентоспособность - экономическая категория, которая может рассматриваться на нескольких уровнях, а именно, конкурентоспособность отраслей, страны, товаропроизводителей, товаров. Между вышеперечисленными уровнями имеется взаимосвязь: в конечном итоге возможность конкретных производителей выпускать конкурентоспособные товары определяет страновую и отраслевую конкурентоспособность.

Конкурентоспособность предприятия - числовая характеристика, на основе которой за определенный период времени оцениваются достигнутые предприятием итоговые результаты его деятельности. Конкурентоспособность предприятия обеспечивается благодаря приобретаемым им разного рода 
преимуществам отличным от основных конкурентов, а именно: инвестиционным, кадровым, имиджевым, финансовым, экономическим и т.п.

Подход к принятию управленческих решений, основывающийся на рассматривании конкурентоспособности продукции как конкурентоспособности предприятия позволяет минимизировать предполагаемые потери и локализовать неблагоприятные ситуации.

В случае, когда у предприятия сформированы устойчивые конкурентные преимущества на длительную перспективу, то данный подход можно отнести к стратегической конкурентоспособности [7].

Ресурсы, являющиеся объектом управления, складываются не только путём привлечения из внешних источников, но также генерируются внутри предприятия, путём создания конкурентных преимуществ, посредством использования ресурсов, поступивших извне, наличие которых определяет эффективность использования материальных и трудовых ресурсов. Такое, более широкое понимание ресурсов, включает в себя понятие «организационные ресурсы», обозначающее средства и возможности, необходимые для выполнения стратегических целей предприятия. Критериями оценки организационных ресурсов как конкурентных преимуществ хозяйственного субъекта являются не столько количественные показатели, сколько такие признаки как ценность, уникальность, воспроизводимость и незаменимость.

Генерирование ресурсов во внутренней среде предприятия, а не только привлечение из внешних источников увеличивает состав функций управления ресурсами, направленных не только на обеспечение эффективной деятельности предприятия, но и формирование конкурентных преимуществ.

Конкурентные преимущества в ресурсах подразумевают под собой:

- привлечение наиболее производительных по сравнению с конкурентами ресурсов;

- создание и владение уникальными ограниченными ресурсами, отсутствующим у других участников рынка;

- создание системы ресурсов, дающая возможность высокой результативности.

Для традиционного затратного подхода к управлению, необходима реализация функций как:

- мобилизация ресурсов;

- формирование конкурентных возможностей и ключевых компетенций;

- совершенствование организационных ресурсов и укрепление ключевых позиций.

Подводя итог рассмотрению влияния привлечения ресурсов на способность предприятия выживать и быть успешным в конкурентной среде, следует подчеркнуть, что большинство хозяйствующих субъектов в настоящее время испытывают затруднения с тем, чтобы эффективно развиваться только за счет собственных ресурсов. Следовательно, они вынуждены все активнее обращаться к внешним источникам, которые дают возможность получать экономические ресурсы на привлекательных условиях. 
Конкуренция заставляет постоянно искать и использовать в производстве новые возможности, требовать совершенствование техники и технологий, повышать качество продукции, снижать затраты, вводить новые формы управления, быстро реагировать на меняющиеся требования и условия рынка и др. Все перечисленное можно отнести к важнейшим преимуществам предприятий.

Особенно актуальными стали оценка и прогноз конкурентоспособности как с точки зрения решения вопроса о конкурентоспособности национальной экономики в целом, так и с точки зрения решения проблем отдельно взятого предприятия.

\section{Список используемых источников:}

1. Веснин В.Р. Стратегия привлечения финансовых ресурсов // Корпоративный менеджмент. URL: http://www.cfin.ru/management/finance/capital/financial_resources.shtml

2. Власов С., Дерюгина Е., Власова Ю. Исследование устойчивости государственных финансов России в краткосрочном и долгосрочном периодах // Вопросы экономики. 2013. № 3. С. 33-49.

3. Годпастер К.Е. Анатомия духовного и социального сознания корпорации: кейс Medtronic, Inc // Российский журнал менеджмента. 2006. T. 4. № 4. С. 99-118.

4. Голованова Н.Б., Горина Т.В. Сравнительная характеристика основных подходов к управлению ресурсами предприятия // Управление экономическими системами.

URL: http://uecs.ru/uecs-72-722014/item/3302-2014-12-30-12-47-45

5. Котлярова В.В. Управленческие решения, направленные на привлечение экономических ресурсов для развития промышленного предприятия // Известия Санкт-Петербургского университета экономики и финансов. 2011. № 4. С. 83-85.

6. Основы теоретической экономики. СПб.: Питер, 2014. 512 c.

7. Солдатов В. Ресурсы - главный фактор результативности и эффективности.

URL: http://www.vsoldatov. com/2010/01/blog-post.html

8. Харламов А.В., Харламова Т.Л. Управление развитием предприятия и минимизация рисков привлечения внешних ресурсов // Проблемы экономики и управления в торговле и промышленности. 2014. № 3(7). C. 22-26.

9. Харламова Т.Л., Бабкина Н.И. Глобализация и производственная активность предприятий (на примере Санкт-Петербурга) // Научно-технические ведомости Санкт-Петербургского государственного политехнического университета. 2009. № 6-1(90). С. 86-93.

(C) 2015, Мясников E.C.

Конкурентоспособность предприятия и проблема привлечения экономических ресурсов
(C) 2015, Myasnikov E.S.

The competitiveness of businesses and the problem of attracting economic resources 